\title{
Zur Geschlechterquote II
}

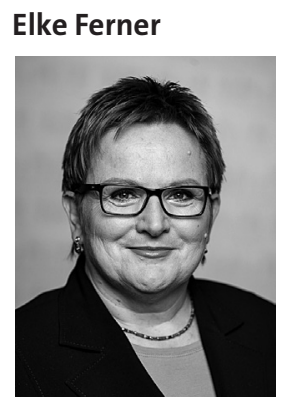

Parlamentarische Staatssekretärin im Bundesministerium für Familie, Senioren, Frauen und Jugend Foto: Bundesregierung/ Denzel
„Mit Parité mehr Frauen in die Parlamente“, das klingt aktuell und zeitgemäß.

Und auf den ersten Blick könnte man denken, wir sind auf einem guten Weg dahin: Unser Land wird von einer Frau regiert. Das Bundesfrauenministerium, in dem ich als Parlamentarische Staatsekretärin bin, sowie vier weitere Ressorts der Bundesregierung werden von einer Frau geführt. Der Anteil der parlamentarischen Staatssekretärinnen in der Bundesregierung ist so hoch wie nie zuvor. Und: der Anteil an Parlamentarierinnen im Deutschen Bundestag ist mit 36,4 Prozent ebenfalls so hoch wie nie zuvor in der deutschen Geschichte. Aktuell haben wir vier Ministerpräsidentinnen. Aber eben nur vier von 16 - und seit Beginn der Bundesrepublik waren es bis jetzt insgesamt nur fünf! In den Landeskabinetten gibt es neben den Regierungschefinnen viele Landesministerinnen. Der Anteil der weiblichen Abgeordneten in den jeweiligen Parlamenten ist dem im Bund zumindest ähnlich.

Das heißt, Frauen sind auf Bundes- und Landesebene inzwischen weit häufiger an der Macht, sie sind sichtbar in der politischen Landschaft.

Trotz dieser Fortschritte ist die politische und gesellschaftliche Repräsentation von Frauen, die immerhin über 50 Prozent der Bevölkerung stellen, insgesamt immer noch unzureichend. Es bleibt also noch viel zu tun, wenn wir eine adäquate Repräsentation auf allen Ebenen erreichen wollen:

Noch nie gab es eine Bundespräsidentin. Erst einmal gab es eine Präsidentin des Bundesverfassungsgerichtes. Die Zahl der Oberbürgermeisterinnen, Bürgermeisterinnen und Landrätinnen ist gering und in den Räten sieht es außerhalb der Großstädte immer noch wie in den 60er und 70er Jahren des letzten Jahrhunderts aus.

Zu meinem Verständnis von Demokratie, Gerechtigkeit und Partizipation gehört mehr als das, was wir haben! Unsere Gesellschaft kann und darf es sich nicht erlauben, auf gut die Hälfte ihres Potentials zu verzichten - gerade nicht in der Politik.

Frauen und Männer müssen überall gleichberechtigt sein - nicht nur in Führungspositionen in der Bundes- bzw. Landespolitik.

Das herrschende Ungleichgewicht zeigt sich unter anderem in der Kommunalpolitik, wo Frauen nach wie vor stark unterrepräsentiert sind: Dort ist nur ein Viertel der ehrenamtlichen Mandate in den Stadträten und Kreistagen von Frauen besetzt.

Noch schlimmer sieht es bei den Führungspositionen auf kommunaler Ebene aus:

96 Prozent der hauptamtlichen Bürgermeister sind männlich,
94 Prozent der Landräte

und 85 Prozent der Oberbürgermeister. ${ }^{1}$

Bei festgefahrenen Strukturen und tradierten Rollenmodellen haben es politisch interessierte Frauen in den Kommunen besonders schwer, den Einstieg in ein Amt mit Verantwortung zu finden. Dennoch nehmen viele die Herausforderung, die eigene Kommune aktiv mitzugestalten und zu verändern, gerne an.

Im Koalitionsvertrag haben wir deshalb die Förderung und Weiterentwicklung des 2009 vom Bundesfrauenministerium ins Leben gerufenen „Helene Weber Kollegs“ vereinbart, der ersten bundesweiten und parteiübergreifenden Plattform für engagierte Frauen in der Politik. In diesem Zusammenhang wird auch der Helene Weber Preis für besonders engagierte Kommunalpolitikerinnen erneut vergeben. Ziel des Helene Weber Kollegs ist es, eine höhere Repräsentanz von Frauen in der Politik zu erreichen und einer Vielzahl hervorragend qualifizierter Frauen den Weg in die politisch entscheidenden Gremien zu ebnen. Denn nur mit einem ausgeglichenen Geschlechtermix in den Gremien und Führungsteams entsteht mehr Vielfalt in der politischen Landschaft, die die Gesellschaft als Ganzes bereichert und uns bei politischen Prozessen vorankommen lässt. Das Helene Weber Kolleg trägt schon jetzt erfolgreich dazu bei, die Einstiegs- und Aufstiegschancen für Frauen in der Politik zu verbessern und die Kooperation untereinander zu fördern.

Mentoringprojekte - egal ob im Rahmen des Helene Weber-Kollegs oder ob in den Parteien und Gewerkschaften - sind hierbei ein zentrales Element. Bei den Mentorings geben bereits etablierte Politikerinnen oder Expertinnen ihr Erfahrungswissen an Neueinsteigerinnen weiter und arbeiten gemeinsam über einen längeren Zeitraum zusammen. Dies ist ein Schlüssel für das Weiterkommen, für die Netzwerkbildung, für den Erfolg von Kommunalpolitikerinnen.

Mentoring alleine reicht aber nicht.

Um tatsächliche Gleichstellung in der Politik zu erreichen, kommen wir nach meiner Überzeugung an verbindlichen Quoten zur Steigerung des Frauenanteils auf verschieden Ebenen nicht vorbei. Quote - das heißt für mich „Chancengleichheit für alle “ und eben nicht Bevorzugung von einigen wenigen. Und da die Quote Frauen und Männern den gleichen Zugang zu politischen Ämtern eröffnet, transportiert sie schließlich auch einen neuen Politikstil in die Gesellschaft. Und von den Erfahrungen ebenso wie vom Engagement

1 Kletzing, Uta/Lukoschat, Helga: „Engagiert vor Ort Wege und Erfahrungen von Kommunalpolitikerinnen“, BMFSFJ, Berlin 2010. 
der Frauen profitiert letztlich nicht nur die Politik, sondern die gesamte Gesellschaft.

Frau Bundesministerin Schwesig hat daher gemeinsam mit ihrem Kollegen Heiko Maas den Entwurf für ein Gesetz zur gleichberechtigten Teilhabe von Frauen und Männern an Führungspositionen in der Privatwirtschaft und im Öffentlichen Dienst erarbeiten lassen.

Wir brauchen die gleichberechtigte Teilhabe der Geschlechter hinsichtlich der Entscheidungsgewalt in öffentlichen und privaten Institutionen und Gremien, in Führungspositionen von Unternehmen, in den Medien und der Zivilgesellschaft, auch der Wissenschaft, in den Familien und Gemeinschaften und natürlich in nationalen, regionalen wie kommunalen Parlamenten.

In diesem Jahr fanden elf Kommunalwahlen statt. Der Frauenanteil in den Kommunalparlamenten hat sich jedoch immer noch nicht nachhaltig verbessert. Selbst parteiinterne Quoten führen auch bei korrekter Anwendung dann nicht zum gewünschten
Ergebnis, wenn das Kommunalwahlrecht Elemente des Persönlichkeitswahlrechts oder regionale Listen enthält. Das Ziel der paritätischen Teilhabe und Repräsentanz beider Geschlechter in politischen Ämtern kommt nicht von allein.

Ohne Wahlrechtsänderung und wirksame Sanktionen wird dieses Ziel nicht zu erreichen sein. Es ist nicht die Frage, ob, sondern wie das Ziel der Parität erreicht werden kann. Das ist keine rein juristische, sondern vor allem eine politische Frage.

Andere Länder machen es uns mit Erfolg vor, wie es geht, beispielsweise Frankreich oder Belgien aber auch Ruanda oder Tunesien. Dort ist es gelungen, entweder beim Demokratieaufbau nach politischen Umbrüchen oder als nachträgliche Wahlgesetzänderung, Regelungen zur paritätischen Repräsentation der Geschlechter zu etablieren.

Es zeigt sich: Ein gleichberechtigtes Wahlrecht ist der elementare Baustein für eine gleichberechtigte Teilhabe am politischen Leben.

\section{Baden-Württemberg}

\section{Der Wechsel beginnt - nur nicht bei der Parité Enttäuschte Erwartungen oder die endlose Geschichte einer Verhinderungsstrategie}

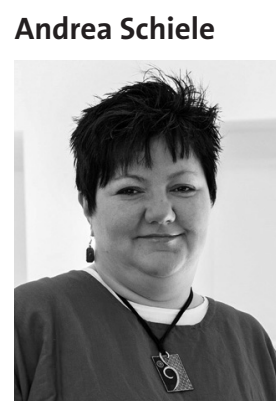

Stellvertretende Vorsitzende der Arbeitsgemeinschaft sozialdemokratischer Frauen (AsF) Baden-Württemberg

\section{Vorausgeschickt}

Unter dem Titel „Der Wechsel beginnt“ vereinbarte die grün-rote Landesregierung in Baden-Württemberg im Mai 2012 einen Koalitionsvertrag, in dem es auf Seite 45 heißt:

„Chancengleichheit von Frauen und Männern

Mehr als die Hälfte der Bevölkerung in BadenWürttemberg sind Frauen und Mädchen. Die neue Landesregierung wird sich in allen Politikfeldern für sie einsetzen und geschlechtsspezifische Benachteiligungen abbauen. Unser Ziel ist ein selbstbestimmtes und partnerschaftliches Miteinander von Frauen und Männern, Mädchen und Jungen in allen Lebensbereichen....

Nach der Landtagswahl ist der Anteil der Frauen nochmals auf nur noch 18 \% (aktuell 2014: 19,2\%) gesunken. Um dies in Zukunft zu ändern, wollen wir sowohl das kommunale Wahlrecht als auch das Landtagswahlrecht dahingehend überprüfen, wie wir es geschlechtergerechter ausgestalten können.“

Soweit, so (scheinbar) gut, die Frauenverbände, allen voran der Landesfrauenrat, gingen nun davon aus, dass es nur eine Frage der Zeit sei, bis die „rote Laterne“ des Frauenanteils in Länderparlamenten durch eine fortschrittliche Änderung des Wahlgesetzes in ein Listenwahlrecht ${ }^{1}$ abgegeben werden kann.

In dieser Zeit wurden von beiden Regierungsparteien eine Resolution des Landesausschusses (Bündnis 90/Die Grünen) bzw. ein Landesparteitagsbeschluss (SPD) verabschiedet, die die Parité für die Listen im
Wahlrecht forderten. Doch in beiden Fraktionen fand dies keine Resonanz.

\section{Die „Reform“ des Kommunalwahlrechts}

Nachdem sich der Widerstand in den Fraktionen zu einer qualitativen Änderung des Wahlrechts früh abzeichnete, startete der Landesfrauenrat 2012 eine Kampagne zur Kommunalwahl 2014 mit dem Titel „Halbe Kraft reicht nicht! Parité in die Parlamente!“. Obwohl es hier bei den Landtagsfraktionen noch in keiner Weise um den eigenen Machterhalt ging, war der Widerstand heftig. Von Gerüchten, dass das Landtagswahlrecht nur mit einer Zweidrittelmehrheit zu ändern sei, über die Bildung einer interfraktionellen Arbeitsgruppe aller Fraktionen, in der man sich verständigte, dass das Landtagswahlrecht nur einvernehmlich geändert werde (nächstes Gerücht: Wahlrechtsreformen seien in Baden-Württemberg immer schon nur einvernehmlich durchgeführt worden), bis hin zur Androhung einer Verfassungsklage falls es die Einführung eines Paritégesetzes zur Kommunalwahl

1 Baden-Württemberg hat als einziges Bundesland kein Listenwahlrecht, sondern ein Wahlsystem aus einer Verbindung von Verhältniswahl und Persönlichkeitswahl: Das Sitzverhältnis der Parteien im Landtag richtet sich nach dem Stimmenverhältnis der Parteien im Land (Verhältniswahl). Die Zuteilung dieser Mandate an die einzelnen Bewerber richtet sich nach den Stimmen, die diese in ihrem jeweiligen Wahlkreis erzielt haben (Persönlichkeitswahl). Es gibt nur Wahlkreisbewerber. Jeder Kandidat und jede Kandidatin muss sich also in einem der 70 Wahlkreise des Landes zur Wahl stellen. 